\title{
Uncertainty studies of topographical measurements on steel surface corrosion by 3D
} scanning electron microscopy

K.W. Kang ${ }^{1, *}$, M.D. Pereda ${ }^{1}$, M.E. Canafoglia ${ }^{2,3}$, P. Bilmes ${ }^{1}$, C. Llorente ${ }^{1,4}$, R. Bonetto ${ }^{5}$

${ }^{1}$ Laboratorio de Investigaciones de Metalurgia Física (LIMF), Facultad de Ingeniería, UNLP, Calle 1 y 47, La Plata, Argentina.

${ }^{2}$ Centro de Química Inorgánica (CEQUINOR), Facultad de Ciencias Exactas, UNLP, La Plata, Argentina.

${ }^{3}$ Facultad de Ciencias Naturales y Museo, UNLP, La Plata, Argentina.

${ }^{4}$ Comisión de Investigaciones Científicas de la Provincia de Buenos Aires - CICPBA.

${ }^{5}$ Centro de Investigación y Desarrollo en Ciencias Aplicadas "Dr. Jorge J. Ronco", CINDECA (CONICET), Facultad de Ingeniería y de Ciencias Exactas, UNLP, La Plata, Argentina.

E-mail address: adriana.kang@ing.unlp.edu.ar (K.W. Kang)

\begin{abstract}
Pitting corrosion is a damage mechanism quite serious and dangerous in both carbon steel boiler tubes for power plants which are vital to most industries and stainless steels for orthopedic human implants whose demand, due to the increase of life expectation and rate of traffic accidents, has sharply increased. Reliable methods to characterize this kind of damage are becoming increasingly necessary, when trying to evaluate the advance of damage and to establish the best procedures for component inspection in order to determine remaining lives and failure mitigation.
\end{abstract}


A study about the uncertainties on the topographies of corrosion pits from 3D SEM images, obtained at low magnifications (where errors are greater) and different stage tilt angles was carried out by using an in-house software previously developed. Additionally, measurements of pit depths on biomaterial surfaces, subjected to two different surface treatments on stainless steels, were carried out. The different depth distributions observed were in agreement with electrochemical measurements.

Keywords: Biomedical devices, 3D SEM, stereo pair, corrosion pit, height maps, uncertainty.

\section{1) Introduction}

The Scanning Electron Microscope (SEM) is largely used for materials characterization due to its great versatility, large depth of field and high lateral resolution. It is well known that the changes in gray levels on SEM images are not related to changes in local height but in the slope. The image brightness can also be affected by the enhanced emission from edges and ridges, effects of surface contaminations such as local oxidation, local variations of composition, detector position, electric and magnetic properties, among others. Thus, the parameters to characterize a surface, by only using a single image, are closer to the image texture than the surface roughness. Nevertheless, these parameters can be used to quantify surface differences of samples subjected to different processes.

In modern applications the exact position of studied objects in space or topographic information about the specimen is required; therefore, the coordinates in all three dimensions are necessary. 
The $3 \mathrm{D}$ reconstruction methods of surface topography, necessary to obtain roughness parameters, can be divided into two principal categories:

1) Stereoscopy, in which a SEM image stereo pair is used. The stereo pair can be obtained by deflecting the electron beam, but it is generally implemented by tilting the specimen stage (Lane, 1969, 1972; Stampfl et al., 1996; Davies and Randle, 2001; Huang et al., 2004; Bonetto et al., 2006; Ponz et al., 2006; Jahnisch and Fatikow, 2007; Marinello et al., 2008; Ostadi et al., 2009; Malboubi et al.,2009; Fatikow et al., 2009; Azevedo and Marques, 2010; Chen et al, 2010; Ostadi et al., 2010). Both, observation of 3D images and measurement of 3D height data are possible. Particularly, by overlapping the stereo pair images, which are one in red and the other in blue or cyan, for example, it is possible to build an anaglyph image which produces a depth effect when glasses with one red lens and the other blue or cyan are used. Practically in all scientific areas, the anaglyph images were usually used as a complementary investigation technique, allowing a more comprehensive study about morphology of the samples searched with SEM (see Hortolà, 2009 for a recent example).

The principal problem of the stereometric method is that this cannot be applied to very smooth surfaces lacking distinguishable details.

2) Shape from shading method which was used first to obtain a surface height image from just a single bidimensional image of an object light-illuminated (Ikeuchi and Horn, 1981) and then, implemented for SEM images, in different versions including one or several detectors (Walker et al., 2005; Pintus et al., 2005; Drzazga et al., 2006; Pintus et al., 2008; Paluszynski and Slowko, 2008; Wzorek et al., 2009, 2010; Vynnyk et al., 2010). The principal disadvantage of the shape from shading method (based on Lambert's angular distribution of the secondary and backscattered electrons), is that the angular distribution is 
far from Lambert's law in the real cases, where the samples have different local orientations concerning the incident electron beam, requiring in many cases, several detectors to obtain images from different orientations. In this work, the first method will be used.

When two images are obtained under different perspectives like in the stereo pair, surface features of different heights differ in their lateral displacement (parallax or disparity) and relative heights ( $\mathrm{z}$ coordinate) can be calculated for each image pixel by using the corresponding disparity value.

In a previous work (Ponz et al., 2006), the EZEImage program was developed to obtain height maps from SEM images. In this software, the Sun (2002) method to find the disparity map, which uses fast cross correlation and two-stage dynamic programming, was implemented. It works on epipolar rectified stereo images so the matching points lie on the same image scanlines of the stereo pair. This means that the tilt axis on the image must be exactly vertical (y axis) and the image center must be the eucentric point.

The equation to find the height values $z(i, j)$ corresponding to each pixel $(i, j)$, measured with respect to a plane that contains the tilt axis and forms an $\left(90-\phi_{1}\right)$ angle with the optical axis is the following (Ponz et al., 2006):

$$
z(i, j)=\frac{\left(x_{1} / M\right)\left(\left(x_{i}-\Delta x\right) \sin \phi_{2} / W M+\cos \phi_{2}\right)-\left(\left(x_{1}-\Delta x\right) / M\right)\left(x_{1} \sin \phi_{1} / W M+\cos \phi_{1}\right)}{\left[\left(1+x_{1}\left(x_{1}-\Delta x\right) /(W M)^{2}\right) \sin \left(\phi_{2}-\phi_{1}\right)+\Delta x \cos \left(\phi_{2}-\phi_{1}\right) / W M\right]}
$$

Where $W$ and $M$ are the working distance and the magnification, respectively and they are equal for both images during eucentric tilting, $\phi_{1}$ and $\phi_{2}$ are the tilt angles corresponding to the left and right images, respectively, $x_{1}$ is the pixel position $(i, j)$ whose height value needs 
to be known on the left image, $\Delta x$ is the disparity and $x_{1}-\Delta x$ is the pixel position of the same point on the right image (measured in the epipolar and by taking the image center as coordinate origin). The $x_{1}$ and $\Delta x$ parameters are measured in the same units as $W$. The $z(i, j)$ expression in Ponz et al. paper (2006) is wrong because the denominator should not be squared.

Equation 1 is the Lane $(1969,1972)$ general equation adapted for eucentrically tilted stereo pairs. When the specimen is tilted $\pm \Delta \phi$ around a normal axis to the beam, it can be written as:

$$
z(i, j)=\frac{2\left(x_{1} / M\right)\left(x_{1}-\Delta x\right) \sin \Delta \phi / M W+\Delta x \cos \Delta \phi / M}{\left[\left(1+\left(x_{1} / M\right)\left(x_{1}-\Delta x\right) / M W^{2}\right) \sin 2 \Delta \phi+\Delta x \cos 2 \Delta \phi / M W\right]}
$$

Besides the independent variables $W, \Delta \phi, x_{1}$ and $\Delta x$, there is a number of additional variables that influence the quality of the reconstruction such as: sample tilt eucentricity, magnification, software algorithm robustness, sharpness of the stereo pair images, among others (Marinello, 2008). Bariani et al (2005) presented a theoretical model regarding the uncertainty calculation of the vertical elevation of a single point, which depends mainly on the tilt angle accuracy and the magnification calibration. They showed that the experimental deviations from the nominal height values confirmed the trend predicted by their model, where the following expression for the variance of the vertical elevation measurement was used:

$$
u_{z}^{2}=\left(\frac{\partial z}{\partial \Delta x}\right)^{2} u^{2}(\Delta x)+\left(\frac{\partial z}{\partial \Delta \phi}\right)^{2} u^{2}(\Delta \phi)
$$


With the variance on the parallax, $u^{2}(\Delta x)$ following the expression:

$$
u^{2}(\Delta x)=\frac{s^{4}}{12}+\Delta x^{2} \frac{u^{2}(s)}{s^{2}}+\Delta x \frac{s^{2}}{\sqrt{3}} \frac{u(s)}{s}
$$

Where $\mathrm{s}$ is the single pixel dimension and $u(s)$ is its uncertainty. Bariani et al. (2005) calibrated the tilt angle in their microscope by means of a laser interferometer system and they obtained 10 arcseconds as tilt angle residual error in their measured samples. Also, they calibrated the magnification and found a pixel size relative error of $1.9 \%$ and $0.81 \%$ in the $100 \mathrm{X}$ and $400 \mathrm{X}$ magnification cases, respectively.

Marinello et al. (2008) searched the critical factors in 3D stereo microscopy by using a galena crystal and commercial software. They found that it is possible to obtain deviations from reference height values (a $22.95 \mu \mathrm{m}$ galena step) within $5 \%$ of the total step height, in ideal conditions, i.e., with pixel size and stage calibrated, magnification in the interval 1000X-3000X and no deviations from the eucentricity condition, while a 30\% error could be expected out of these optimum conditions.

In a previous paper (Ponz et al, 2006) it was stated that due to the fact that the disparity is an integer number on a digital image, the subpixel resolution implemented in the EZEImage program (which allows to increase the precision in the height difference values, reaching an equal or smaller value than lateral resolution depending on the $\Delta \phi$ angle), is valid only in the epipolar axis and on the "plateaus" with quasi constant disparity values. Therefore, and taking into account that a smoothing method on the disparity values between two plateaus has not been implemented in this software, the estimated maximum error of 
the $\Delta x$ disparity will be 1 pixel in microns, i.e. $u_{\max }(\Delta x)=122 / M[\mu \mathrm{m}]$ for the microscope used here.

In this paper, a study about uncertainties in the corrosion pit topography in metallic samples, by using EZEImage program and a Philips SEM 505 microscope, was carried out. Carbon steel samples of a boiler tube from a power thermoelectric generator were used to search these uncertainties under different experimental conditions. Reliable methods to characterize corrosion pits are becoming increasingly necessary in any type of corroded samples (API 570, 1988; API 579 and API 581, 2000), and particularly, in stainless steel samples for orthopedic implants, since this damage mechanism is very common and dangerous in these biomaterial devices (Pohler, 1986; Choules et al., 2009; Tiansheng Wang et al., 2006). Therefore, the corrosion pit depth distributions on samples under different surface treatments to be used as implants were analyzed. The results obtained are compared with electrochemical test.

\section{Materials and Methods}

\subsection{Instrumental}

A scanning electron microscope Philips SEM 505 was used, equipped with a digital scanning interface ADA II and a Scandium SIS Image Analysis software of the Microscopy Laboratory of Centro de Investigación y Desarrollo en Ciencias Aplicadas “Dr. Jorge J. Ronco"(CINDECA) .

In this microscope tilt axis position was calibrated resulting not normal to the optical axis but presenting a rotation of $12^{\circ}$ clockwise. The EZEImage software (Ponz et al., 2006) was 
used to obtain dense height maps. In this software, the tilt axis on the images must be in the same direction as the optical axis (y axis), therefore, the images were rotated $102^{\circ}$ counterclockwise.

\subsection{Image rectification}

An important condition to be considered in the acquisition of stereo pairs is to maintain the eucentric point in the image center when EZEImage software is used. Therefore, those few images that did not satisfy this condition were rectified, so the position of the texture corresponding to the left image center was the same as that on the right image center. Finally, centered areas of $600 \times 700$ pixels of the two pair images were obtained and used in the calculation of height values.

\subsection{Numerical analysis of uncertainties for different magnifications}

Since laser interferometer calibration of the tilt angle (Bariani et al., 2005) was not possible, a way to estimate a $u(\Delta \phi)$ maximum value was implemented in this work. This was carried out by studying the variation of the $\alpha$ angle between the normal to the leastsquare mean plane (that better fits all the surface height values) and the normal to the plane which contains the tilt axis. This angle value was obtained from the text file "height" generated by the EZEImage program.

In order to analyze such angular variation, fifteen SEM image stereo pairs were obtained, verifying the stage eucentric position, i.e. the picture in the center of the two pair images 
were the same. The angle variation could be due to either a variation of the stage tilt or to the subpixel variation of the eucentric position. In this study, we associated the change of the angle only to the stage tilt variation. This approximation would yield a maximum value of the tilt error $u(\Delta \phi)$, which, obviously, would be larger than the one obtained by Bariani et al. (2005), which was 10 arcseconds as previously mentioned. Also, as mentioned in the Introduction section, a maximum error $u(\Delta x)= \pm 1$ pixel $[\mu \mathrm{m}]$ was considered due to the fact that the disparity is an integer number on a digital image.

In this way, the relative uncertainties $u_{z} / z$ with these maximum possible errors in $\Delta \phi$ and $s$ were calculated by using eqs. 3 and 4 , at $\Delta \phi= \pm 8^{\circ}$ around a normal axis to the beam and working distance $W=44 \mathrm{~mm}$.

The relative uncertainties $u_{z} / z$ were calculated at $\Delta \phi= \pm 8^{\circ}$ and $W=44 \mathrm{~mm}$ in the ideal conditions (Bariani et al., 2005), i.e., $u(s) / s \approx 0.02$ for magnifications $100 \mathrm{X}$ and $200 \mathrm{X}$; and $u(s) / s \approx 0.01$ for magnification $400 \mathrm{X}$ and $u(\Delta \phi)=10$ arcseconds for the three magnifications. The results are shown in section 3.1.

\subsection{Metallic samples for the study of the height value uncertainties}


Three samples of $400 \mathrm{~mm}^{2}$ area were obtained from a corroded boiler tube piece of a power thermoelectric generator. The oxide layers on their inner surface were removed with hot hydrochloric acid solution as only superficial cleaning treatment.

A preliminary study of the first sample showed several corrosion pits on the surface and particularly one of them on the sample edge. A stereo pair of the edge region (see Fig. 3a in section 3.2.1) was obtained in order to compare pit depths in different places obtained from the 3D values provided by EZEImage, with the same values directly measured on the cross section (Fig. 3 b in section 3.2.1) by using the microscope software.

Taking into account that the matching point method implemented in the EZEImage software requires a relatively smooth local region of the disparity map (Ponz et al., 2006), the edge pixels will not have valid height values. Due to this fact, in a first step, a stereo pair was obtained and in a second step $10 \mu \mathrm{m}$ of the cross section surface were removed with \#1000 emery paper (this magnitude was estimated by means of SEM images before and after material removal). Later on, the sample was again placed in the SEM specimen stage with cross section surface normal to the optical axis, in order to obtain height values for different $(\mathrm{x}, \mathrm{y})$ coordinates of the pit. Thus, having the previous stereo images, the stereo height values corresponding to any point of the sample pit (represented by the pixel on the image) could be compared with the corresponding values measured on the polished sample cross section.

In order to search the possible variations in the height values, when greater magnifications are used, stereo pairs at $\Delta \phi= \pm 8^{\circ}$, of a same pit at $100 \mathrm{X}$ and $400 \mathrm{X}$ magnifications of the second sample, were obtained. 
Finally, in order to study the reliability of the height values in any specimen tilt, stereo pairs at different tilt angle conditions were obtained from the third sample.

\subsection{Study of metallic biomaterial samples}

Samples of $100 \mathrm{~mm}^{2}$ area and 3mm thick from a 316LVM (Low carbon Vacuum Melting) stainless steel plate (ASTM F138 \& F139/ISO 5832-1) were obtained. These stainless steels were chosen because they have an extensive application as biomaterials, particularly for orthopedic implants, as they combine a good biofunctionality with acceptable biocompatibility and low cost.

Six of these samples were subjected to blasting and passivation treatments in different conditions and later under a same corrosion process.

\subsubsection{Surface treatments and electrochemical measurements}

Blasting process was carried out at $3 \mathrm{~kg} / \mathrm{cm}^{2}$ pressure with aluminum silicate particles of 10$150 \mu \mathrm{m}$ size distribution for the samples I to III, and at $5 \mathrm{~kg} / \mathrm{cm}^{2}$ pressure with $100 \mu \mathrm{m}$ average size silica particles in the case of samples IV to VI.

The samples were put into contact with acetone for 30 minutes for cleaning purposes and washed with water, dried on a drying stove and cleaned by ultrasound for 20 minutes in acetone and for 10 minutes in commercial alcohol in a second step. Finally, the samples were washed with commercial alcohol and dried. 
The passivation treatments were carried out at room temperature in solutions of $40 \% \mathrm{v} / \mathrm{v}$ nitric acid for 30 minutes for samples I to III and 20\% v/v nitric acid for $1 \mathrm{~h}$ for samples IV to VI.

In order to study the formation of corrosion pits, cyclic potentiodynamic polarization was performed in a conventional three-electrode cell in Ringer's aqueous solution $(8.6 \mathrm{~g} / \mathrm{L}$ $\mathrm{NaCl}, 0.3 \mathrm{~g} / \mathrm{L} \mathrm{KCl}, 0.33 \mathrm{~g} / \mathrm{L} \mathrm{CaCl}_{2}$ ) deaerated with pre-purified nitrogen for $1 \mathrm{~h}$ at $37^{\circ} \mathrm{C} . \mathrm{A}$ platinum wire was used as the counter-electrode, and a saturated calomel electrode (SCE) was used as the reference electrode. Cyclic potentiodynamic polarization curves were recorded at $50 \mathrm{~V} / \mathrm{h}(13.8 \mathrm{mV} / \mathrm{s})$ from $-1.2 \mathrm{~V}_{\mathrm{SCE}}$ up to $1.2 \mathrm{~V}_{\mathrm{SCE}}$.

\subsubsection{Attainment and processing of SEM image}

Stereo pairs of different regions in both sample sets were obtained. The stereo pairs for samples I to III were obtained at the tilt angle $\Delta \phi= \pm 5^{\circ}$ around a normal axis to the beam and at a magnification $M=100 \mathrm{X}$ while those corresponding to the samples IV to VI were obtained at $\Delta \phi= \pm 8^{\circ}$ and $M=200 \mathrm{X}$. Although the possible biggest tilt angles and magnifications are advisable to minimize errors, lesser values allow studying a greater number of pits on a single SEM image pair. An extra stereo pair with two of the pits of the sample VI was obtained at $\Delta \phi= \pm 5^{\circ}$ and $\mathrm{M}=100 \mathrm{X}$ in order to compare pit depths for the two different superficial conditions used.

\section{Results and discussion}

\subsection{Uncertainty analysis at different magnifications}


The tilt error obtained, as mentioned in section 2.3, provided a value $u(\Delta \phi)= \pm 97$ arcseconds, which was used to obtain the overestimated values of relative uncertainties $u_{z} / z$ shown in Fig. 1a for 100X, 200X and 400X magnifications and $\Delta \phi= \pm 8^{\circ}$. The corresponding values for the ideal conditions can be seen in Fig. $1 \mathrm{~b}$.
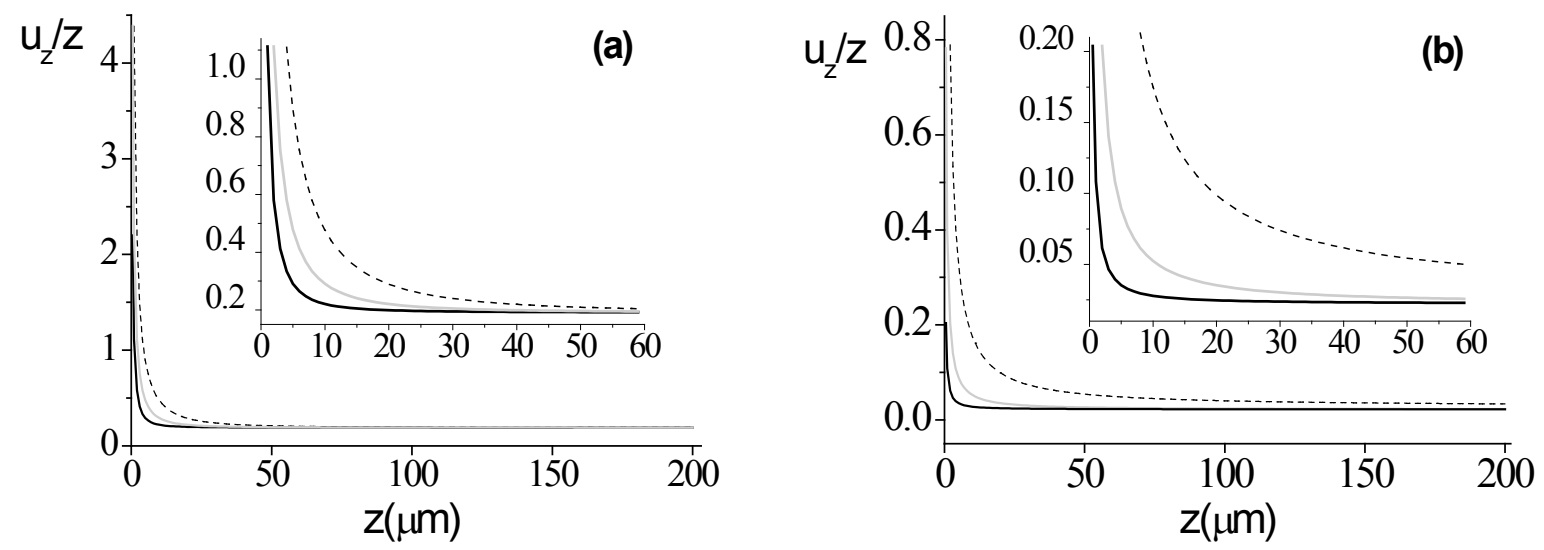

Fig. 1. Relative uncertainties for $\Delta \phi= \pm 8^{\circ}$ and $W=44 \mathrm{~mm}$. Dotted line: 100X, gray line: 200X and black solid line: 400X magnifications. The insets show amplified views of relative uncertainties for low z values; (a) Overestimated $(u(\Delta x)= \pm 1$ pixel $[\mu \mathrm{m}]$ and $u(\Delta \phi)=$ \pm 97 arcseconds); (b) For the ideal conditions $(u(s) / s=0.02$ for $100 \mathrm{X}$ and $200 \mathrm{X}$ magnifications and 0.01 in $400 \mathrm{X}$ case and $u(\Delta \phi)=10$ arcseconds).

Similar calculations for $\Delta \phi= \pm 5^{\circ}$ and $100 \mathrm{X}$ magnification are shown in Fig. 2. 

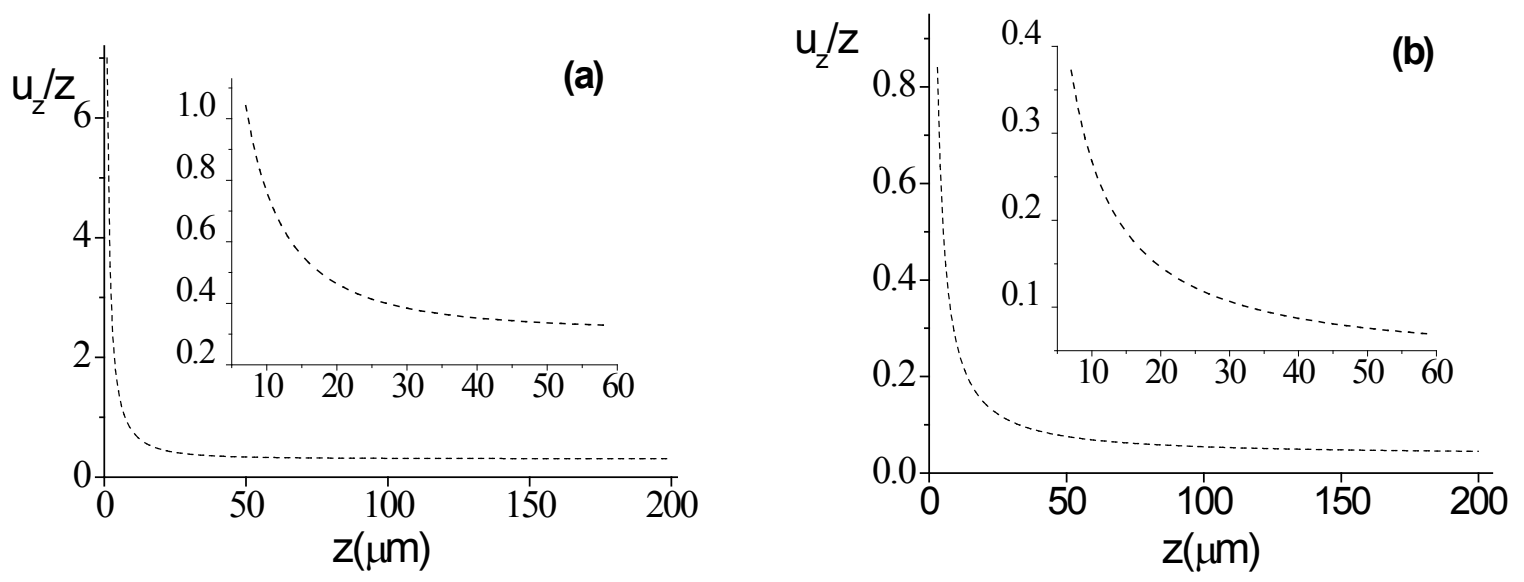

Fig. 2. Relative uncertainties for $\Delta \phi= \pm 5^{\circ}, W=44 \mathrm{~mm}$ and $100 \mathrm{X}$ magnification. (a)

Overestimated $(u(\Delta x)= \pm 1$ pixel $[\mu \mathrm{m}]$ and $u(\Delta \phi)= \pm 97$ arcseconds); (b) For the ideal conditions $(u(s) / s=0.02 \%$ and $u(\Delta \phi)=10$ arcseconds $)$. The insets show amplified views of relative uncertainties for low $\mathrm{z}$ values.

As developed in section 2.3 and observed in Fig. 1b, the relative uncertainties tend to $8 \%$ starting from $30 \mu \mathrm{m}$ height values and to $5 \%$ starting from $60 \mu \mathrm{m}$ when considering a tilt angle $\Delta \phi= \pm 8^{\circ}$, a magnification $M=100 \mathrm{X}$ and ideal conditions in the $\Delta \phi$ and pixel size errors. In the case of $200 \mathrm{X}$ and $400 \mathrm{X}$ magnification, $\Delta z / z$ values less than $5 \%$ were obtained starting from $10 \mu \mathrm{m}$ and $3 \mu \mathrm{m}$ height values respectively. On the other hand, when the uncertainties in $\Delta \phi$ and $\Delta s$ were overestimated, the relative uncertainties close to $30 \mu \mathrm{m}$ increased to $24 \%, 21 \%$ and $20 \%$ at $100 \mathrm{X}, 200 \mathrm{X}$ and $400 \mathrm{X}$ magnifications, respectively. When $\Delta \phi= \pm 5^{\circ}$ and $M=100 \mathrm{X}$ were considered, $u_{z} / z$ values less than $15 \%$ were obtained starting from $20 \mu \mathrm{m}$ height values in ideal conditions, while for $\mathrm{z}$ values close to $30 \mu \mathrm{m}$ the relative uncertainties increased to $38 \%$ when $\Delta \phi$ and $\Delta x$ errors were overestimated. 


\subsection{Metallic samples for the study of the height value uncertainties}

\subsubsection{Verification of reliability of the height values with EZEImage program and Philips SEM 505 by means of the pit cross-section}

Due to the lack of suitable reference calibration standards, the accuracy of the height value calculations in a corrosion pit (Fig. 3a), with EZEImage program and Philips SEM 505 microscope, was tested by means of several cross section measurements of the same pit (Fig. 3b). The height values obtained by means of stereo pairs (at working distance $W=$ $44 \mathrm{~mm}$, tilt angle $\Delta \phi= \pm 8^{\circ}$, and magnification $M=100 \mathrm{X}$ ) and from the cross section (at $M=220 X)$ are summarized in Table 1.
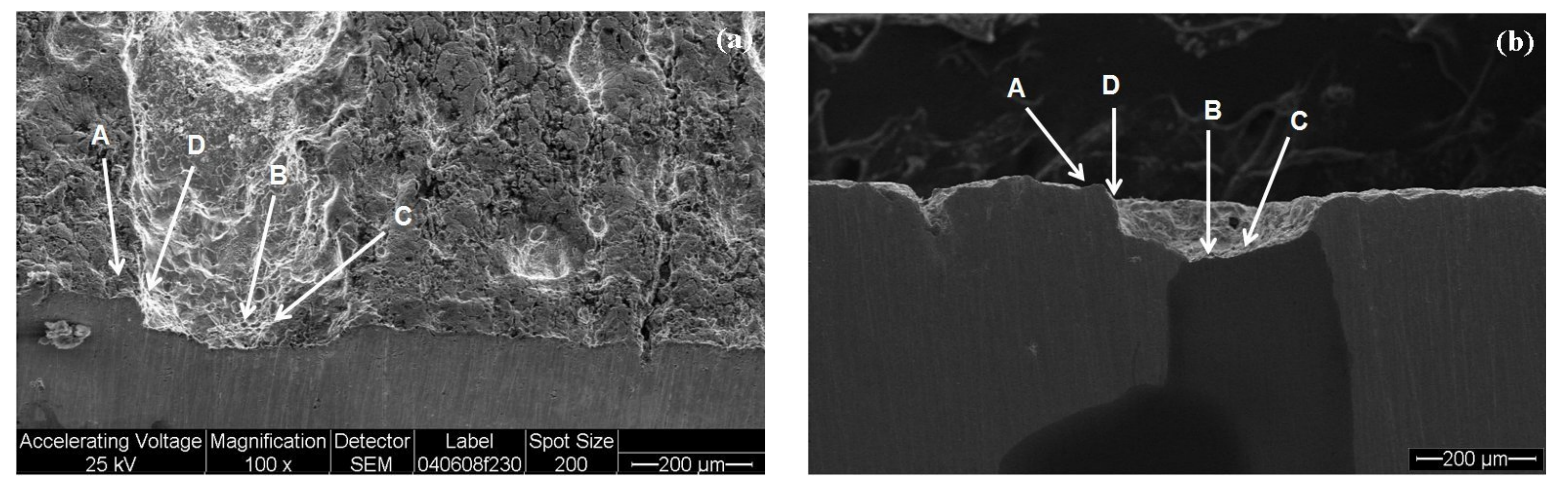

Fig. 3. (a) Pit SEM image obtained at $+8^{\circ}$ tilt angle and $100 \mathrm{X}$ magnification of first

corroded sample; (b) Cross-section of the pit in image (a). The A, B, C and D points in both images are the same as those in Table 1, and represent the coordinates of equivalent measurements obtained by both methods.

The depth data obtained at $100 \mathrm{X}$ magnification are very reliable by considering the estimated minimum error in ideal conditions. As it can be observed in the fifth column in 
Table 1, except for the last point, the difference between the height data obtained by means of stereometry $\left(\Delta \mathrm{H}_{\text {Stereo }}\right)$ and those obtained from the cross section $\left(\Delta \mathrm{H}_{\text {Cross Section }}\right)$, differ within the estimated minimum uncertainty (Fig. 1b).

Table 1. Pit height values measured by EZEImage regarding image center $\left(\mathrm{H}_{\text {Stereo }}\right)$. The third and fourth columns show the corresponding height values (regarding A point) by means of stereo pairs and cross-section of the pit, respectively. In the calculation of the errors in fourth column, eq. 4 was used by assuming $u(s) / s=0.02$.

\begin{tabular}{|c|c|c|c|c|}
\hline $\begin{array}{c}\text { Coordinates } \\
(\mathrm{X}, \mathrm{Y}) \\
{[\text { pixel] }}\end{array}$ & $\begin{array}{l}\mathrm{H}_{\text {Stereo }} \\
{[\mu \mathrm{m}]}\end{array}$ & $\begin{array}{c}\Delta \mathrm{H}_{\text {Stereo }} \\
{[\mu \mathrm{m}]}\end{array}$ & $\begin{array}{c}\Delta \mathrm{H}_{\text {Cross Section }} \\
{[\mu \mathrm{m}]}\end{array}$ & $\mid\left(\Delta \mathrm{H}_{\mathrm{CS}}-\underset{\%}{\left.\Delta \mathrm{H}_{\mathrm{S}}\right)} / \Delta \mathrm{H}_{\mathrm{CS}} \mid\right.$ \\
\hline $605,742(\mathrm{~A})$ & 52.6 & & & \\
\hline $570,592(\mathrm{~B})$ & -74.5 & 127.1 & $124.1 \pm 2.9$ & 2.4 \\
\hline $556,533(\mathrm{C})$ & -61.4 & 114.0 & $112.0 \pm 2.7$ & 1.8 \\
\hline $604,734(\mathrm{D})$ & 30.69 & 21.91 & $22.89 \pm 0.90$ & 1.0 \\
\hline 569,614 & -78.9 & 131.5 & $130.1 \pm 3.0$ & 1.1 \\
\hline 583,673 & -52.6 & 105.2 & $108.4 \pm 2.5$ & 2.9 \\
\hline 584,662 & -56.97 & 109.57 & $108.4 \pm 2.5$ & 1.1 \\
\hline 596,713 & -30.7 & 83.3 & $85.5 \pm 2.1$ & 2.6 \\
\hline 592,692 & -35.1 & 87.7 & $85.5 \pm 2.1$ & 2.6 \\
\hline 623,836 & 74.52 & -21.92 & $20.48 \pm 0.85$ & 7.0 \\
\hline 613,771 & 48.23 & 4.37 & $7.23 \pm 0.59$ & 40 \\
\hline
\end{tabular}




\subsubsection{Data reliability study for two different magnifications.}

In Fig. 4, is shown a pit at $100 \mathrm{X}$ and $400 \mathrm{X}$ magnification of the second corroded sample.

Table 2 shows several height data regarding an arbitrary point (point 1 in the table), obtained from the corresponding stereo pairs for each magnification.
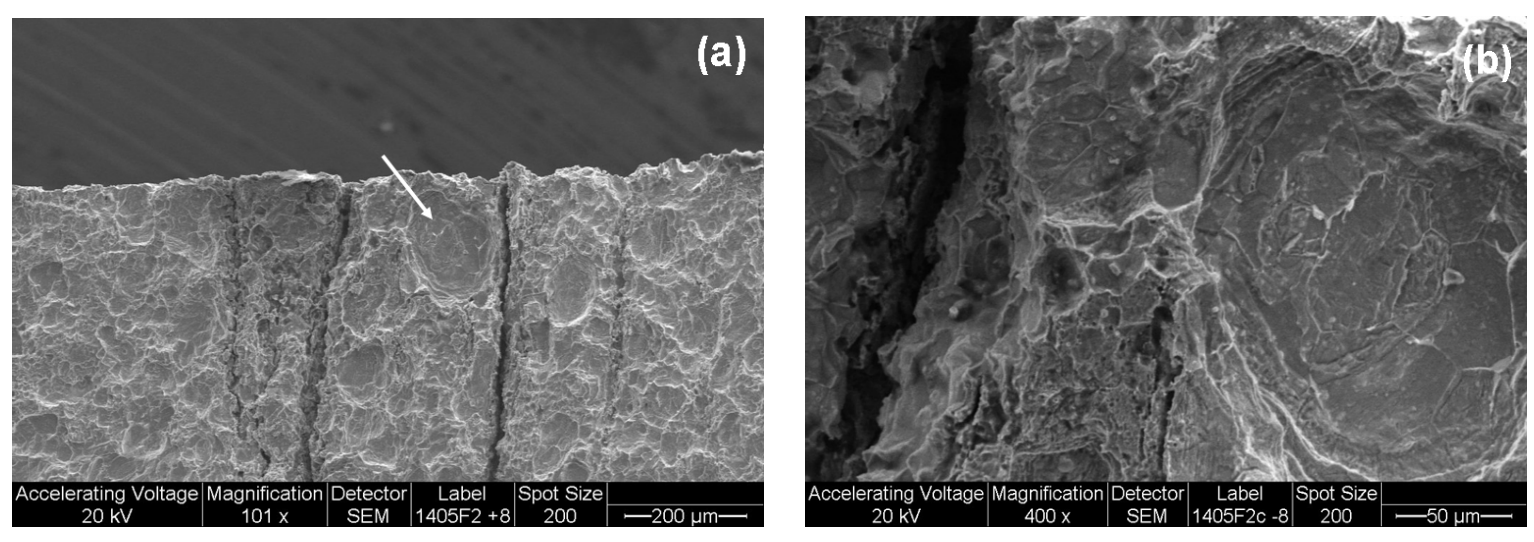

Fig. 4. Micrographs showing pits in the second corroded sample. (a) At 100X

magnification; (b) Pit marked with a white arrow in (a) at 400X.

By considering the estimated maximum errors (Fig. 1a), the depth values obtained at 100X and 400X magnifications (fourth and seventh column in Table 2, respectively) are absolutely equivalent. As can be observed in all cases, the difference between both height values $(\varepsilon \%)$ is less than the minimum between both estimated maximum uncertainties, i.e., those corresponding to $400 \mathrm{X}$ magnification. 
Table 2. Height values $(\Delta \mathrm{H})$ corresponding to the pits shown in Fig. 4, relative to point 1, at two different magnifications; $\varepsilon \%=100 .\left|\left(\Delta \mathrm{H}_{100 \mathrm{x}}-\Delta \mathrm{H}_{400 \mathrm{x}}\right) / \Delta \mathrm{H}_{100 \mathrm{x}}\right|$ in the last column.

\begin{tabular}{cccccccc}
\hline Point & $\begin{array}{c}\text { Coordinates } \\
100 \mathrm{X}\end{array}$ & $\begin{array}{c}\mathrm{H}_{100 \mathrm{X}} \\
{[\mu \mathrm{m}]}\end{array}$ & $\begin{array}{c}\Delta \mathrm{H}_{100 \mathrm{X}} \\
{[\mu \mathrm{m}]}\end{array}$ & $\begin{array}{c}\text { Coordinates } \\
400 \mathrm{X}\end{array}$ & $\begin{array}{c}\mathrm{H}_{400 \mathrm{X}} \\
{[\mu \mathrm{m}]}\end{array}$ & $\begin{array}{c}\Delta \mathrm{H}_{400 \mathrm{X}} \\
{[\mu \mathrm{m}]}\end{array}$ & $\begin{array}{r}\varepsilon \\
\%\end{array}$ \\
\hline 1 & $(418,430)$ & -17.53 & & $(346,448)$ & -15.34 & & \\
2 & $(404,398)$ & -35.1 & -17.5 & $(278,308)$ & -32.9 & -17.5 & 0.0 \\
3 & $(364,406)$ & $3.728 \times 10^{-4}$ & 17.53 & $(108,354)$ & 3.29 & 18.63 & 6.3 \\
4 & $(468,382)$ & -35.1 & -17.5 & $(558,236)$ & -34.0 & -18.6 & 6.3 \\
5 & $(432,424)$ & -4.38 & 13.15 & $(402,420)$ & -3.29 & 12.05 & 8.4 \\
6 & $(416,382)$ & -39.5 & -21.9 & $(336,238)$ & -38.4 & -23.0 & 5.0 \\
7 & $(410,430)$ & -21.9 & -4.4 & $(306,448)$ & -18.63 & -3.3 & 25 \\
\hline
\end{tabular}

\subsubsection{Dependence on height data with different specimen stage tilt conditions}

The most common way to obtain a SEM image is with the specimen tilted away from normal incidence (i.e., $\phi \neq 0$ ), since more secondary electrons escape from the specimen at larger tilt angles. Therefore, it is necessary to verify that the height values obtained with EZEImage are equivalent to any specimen tilt angle.

Dense height maps (where each gray level corresponds to a given height value), with different specimen tilt conditions for left and right images were obtained at magnification $M=100 \mathrm{X}$. 

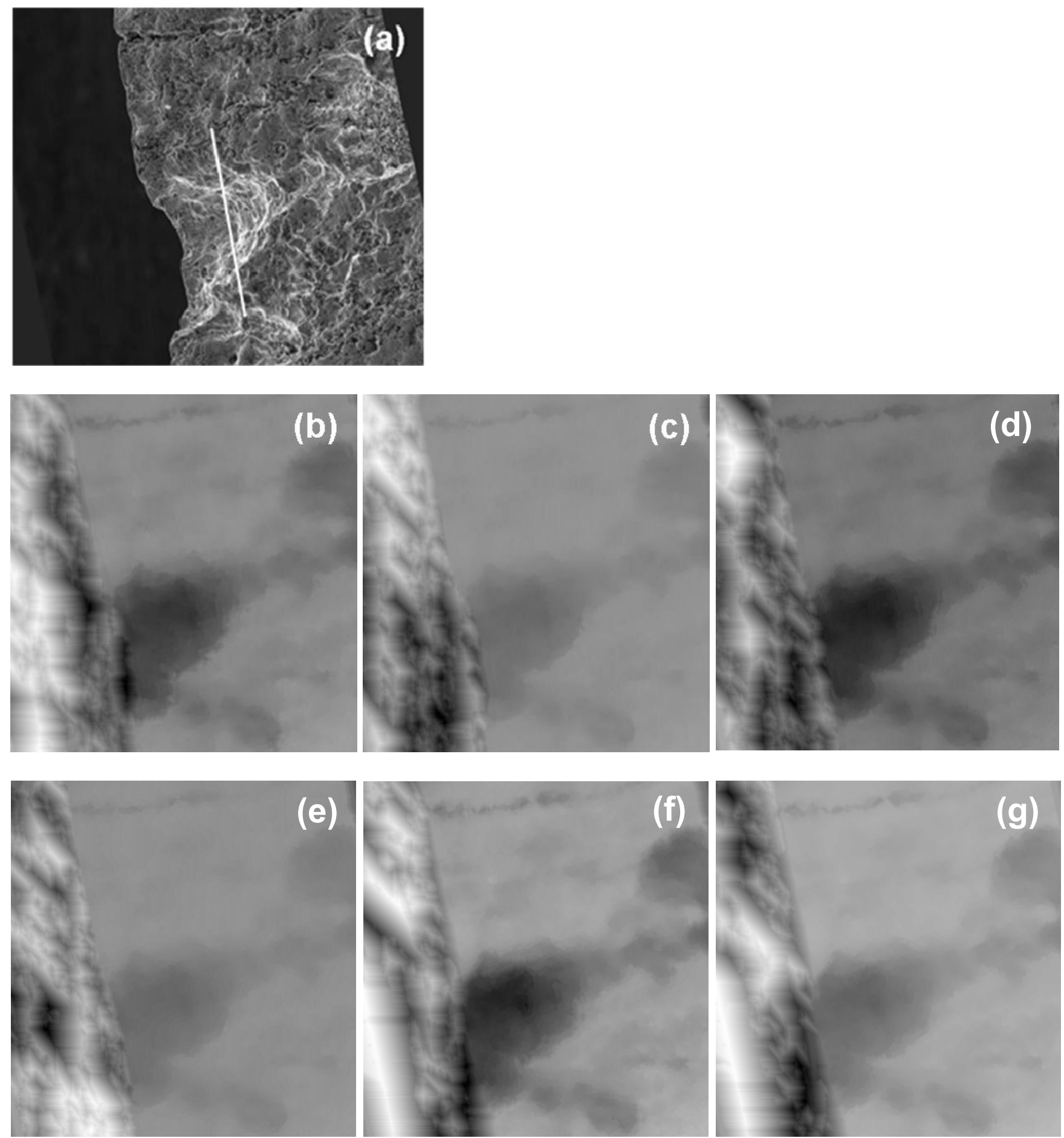

Fig. 5. Corrosion pit of third corroded sample: (a) SEM image obtained at $M=100 \mathrm{X}$ and $\phi_{2}=32^{\circ}$. The straight line corresponds to the deepest pit region where the height profiles 
were calculated; (b)-(g) Dense height maps for stereo pairs at $\left(\phi_{1}, \phi_{2}\right)$ tilt angles equal to: $\left(16^{\circ}, 32^{\circ}\right) ;\left(16^{\circ}, 24^{\circ}\right) ;\left(8^{\circ}, 24^{\circ}\right) ;\left(8^{\circ}, 16^{\circ}\right) ;\left(-8^{\circ}, 16^{\circ}\right)$ and $\left(-8^{\circ}, 8^{\circ}\right)$, respectively

Figure 5a shows a corrosion pit corresponding to the third corroded sample, at $\phi_{2}=32^{\circ}$ and $M=100 \mathrm{X}$. Dense height maps for different tilt conditions can be seen in Figures 5b-g. Blurring effects, occurring in the depth discontinuity regions as was mentioned in section 2.4 , can be seen in the regions on the left of each frame.

In Fig. 6, the height profile across the pit for the different tilt conditions is displayed.

Height values $\Delta H$, corresponding to the difference between the averages of the highest and lowest values on the profile are shown in Table 3.

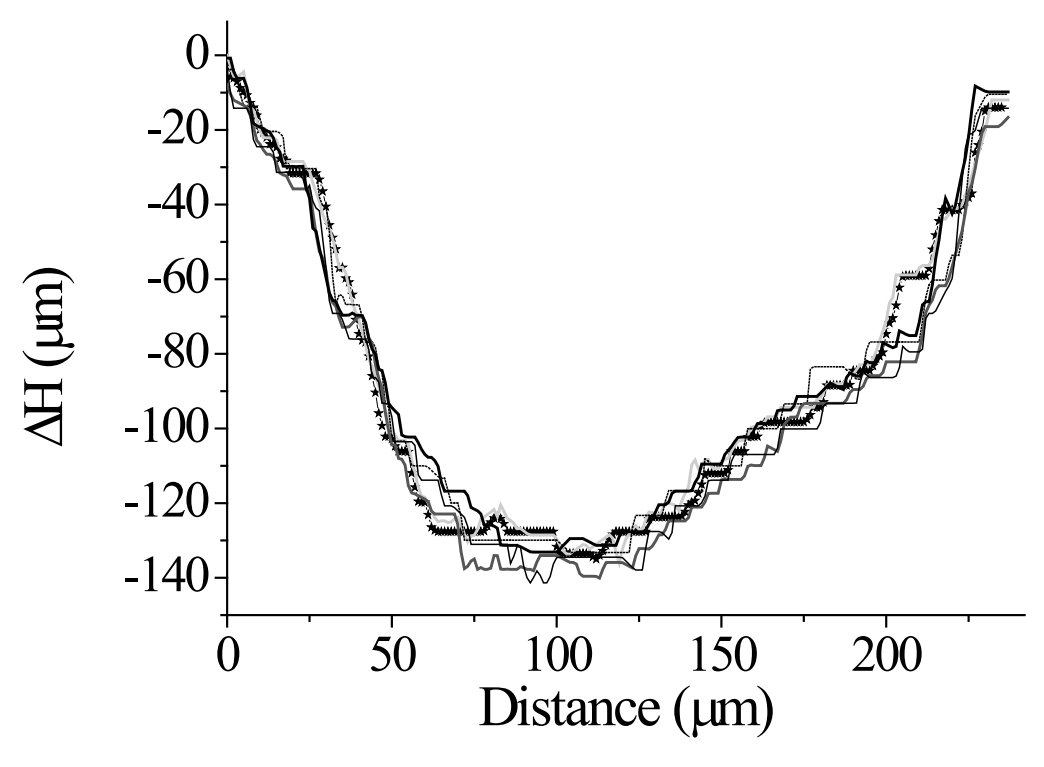

Fig. 6. Height profiles across the pit for the different tilt conditions: $\left(16^{\circ}, 32^{\circ}\right)$ black solid line; $\left(16^{\circ}, 24^{\circ}\right)$ thin black line; $\left(8^{\circ}, 2^{\circ}\right)$ dark gray line; $\left(8^{\circ}, 16^{\circ}\right)$ black dotted line; $\left(-8^{\circ}\right.$, $\left.16^{\circ}\right)$ light gray line and $\left(-8^{\circ}, 8^{\circ}\right)$ line with stars, respectively. 
As can be observed from both Fig. 6 and Table 3, the method of 3D data acquisition and their corresponding processing implemented in EZEImage program is quite reliable to reproduce the pit depth data obtained at different sample tilts, within the estimated minimum error (Fig. 1b). Although the uncertainty in the repeatability of the pit true shape in the sample may be large on any particular point, the calculations of their depths can be obtained with a lesser error, even working at tilt angles lower than $\pm 8^{\circ}$. Obviously, in the case of very small depth measurements it should always be kept in mind that lesser errors will exist when a larger $\Delta \phi$ tilt angle is used. If the whole profile reconstruction is required, larger magnifications and $\Delta \phi$ larger than $8^{\circ}$ will be advisable, in order to minimize errors.

Table 3. Pit depth calculated as the difference between the average of the height values of the plain and the average of the values in the valley from the corresponding height profile, for the different stage tilt conditions.

\begin{tabular}{rc}
\hline$\left(\phi_{1}, \phi_{2}\right)$ & Pit depth $(\mu \mathrm{m})$ \\
\hline$\left(-8^{\circ}, 8^{\circ}\right)$ & 123.2 \\
$\left(-8^{\circ}, 16^{\circ}\right)$ & 123.7 \\
$\left(8^{\circ}, 16^{\circ}\right)$ & 126.6 \\
$\left(8^{\circ}, 24^{\circ}\right)$ & 125.1 \\
$\left(16^{\circ}, 24^{\circ}\right)$ & 122.6 \\
$\left(16^{\circ}, 32^{\circ}\right)$ & 125.7 \\
\hline
\end{tabular}




\subsection{Study of metallic biomaterial samples}

The behavior in the pit depth in the samples I to VI is shown in Fig. 7. In this figure, the pit depth was calculated as the difference between the average of the height values of the plain and the average of the values in the valley from the corresponding height profile.
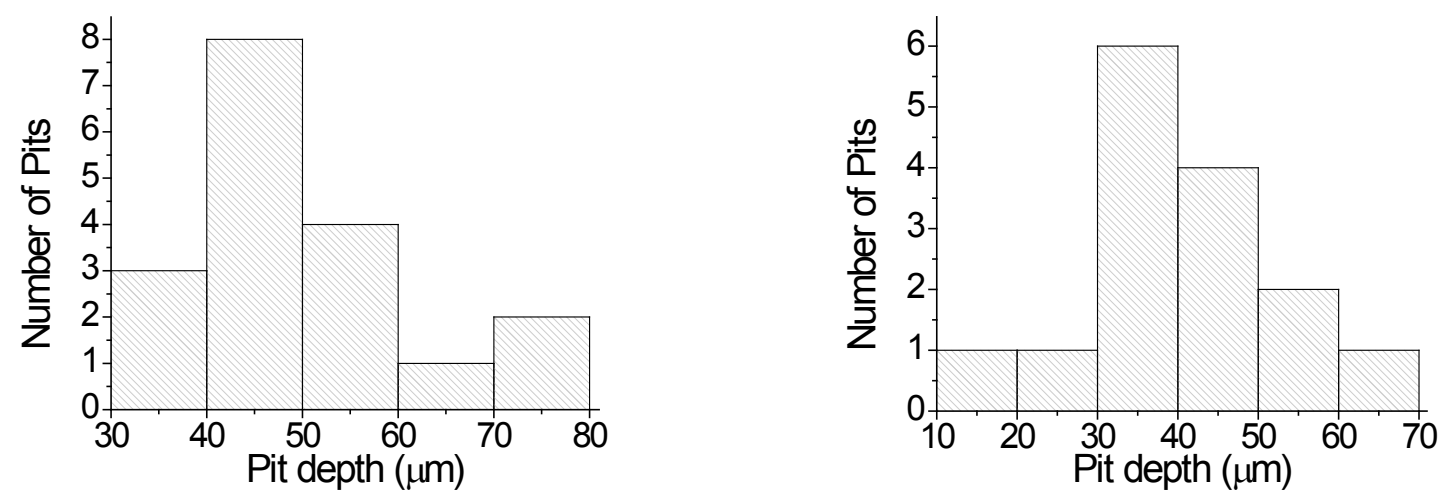

Fig. 7. Pit depth distributions for the samples I to III, with pit average depth $=50( \pm 12) \mu \mathrm{m}$ (left) and samples IV to VI, with pit average depth $=40( \pm 13) \mu \mathrm{m}$ (right).

The average values of the pit diameters showed larger dispersion than those corresponding to their depths, being $83( \pm 32) \mu \mathrm{m}$ for the samples I-III and $96( \pm 31) \mu \mathrm{m}$ for the samples IVVI.

Additionally, a correlation was found between the results shown in Fig. 7 and the analysis of the cyclic polarization curves.

The polarization test showed initiation and propagation of pitting corrosion during the forward scanning and repassivation in the reverse scanning. The pitting potential was 0.563 $\mathrm{V}_{\mathrm{SCE}}$ for samples I to III and $0.672 \mathrm{~V}_{\mathrm{SCE}}$ in the case of samples IV to VI. 
In all corrosion processes carried out by an electrochemical mechanism, the consumption of material in the particular partial reaction is related to the electric current $I(A)$ by Faraday's law (Heitz, 2006):

$m=P I t /(k F)=P Q /(k F)$

where $m$ is the amount of consumed electrochemically material (g), $P$ is the molar weight $\left(\mathrm{g} \mathrm{mol}^{-1}\right), F$ is the Faraday number (96487 $\left.\mathrm{A} \mathrm{s} \mathrm{mol}^{-1}\right), t$ is the time (s), $k$ is the charge number, and $Q$ is the total charge (A s). The value of $Q$ is proportional to the area contained in the hysteresis loop between the repassivation potential and the anodic limit of the test $\left(1.2 V_{S C E}\right)$ (Wilde and Williams, 1971).

The obtained $Q$ values were: 192.5 A-s for samples I-III and 129.03 A-s for samples IV-VI. Therefore, the amount of material consumed in the first sample set was larger than in the second, which correlates well with the pit depths since they were larger for the samples I to III.

This correlation was possible because we assumed that the observed differences in the two histograms of Fig. 7 were due to the different methods applied to the surface treatment (Aparicio et al., 2003; Barranco et al., 2010; Azar et al., 2010) and not to the use of different magnifications and tilt angles in each sample set.

This assertion was also confirmed by studying the depths of two pits in the sample VI at the same conditions as samples I-III, i.e., $100 \mathrm{X}$ magnification and $\Delta \phi= \pm 5^{\circ}$, obtaining $48.51 \mu \mathrm{m}$ at $M=200 \mathrm{X}, \Delta \phi= \pm 8^{\circ}$ and $48.43 \mu \mathrm{m}$ at $M=100 \mathrm{X}, \Delta \phi= \pm 5^{\circ}$ for the first pit, and $47.34 \mu \mathrm{m}$ at $M=200 \mathrm{X}, \Delta \phi= \pm 8^{\circ}$ and $48.41 \mu \mathrm{m}$ at $M=100 \mathrm{X}, \Delta \phi= \pm 5^{\circ}$ for the second pit. The results 
showed that the relative difference between both depths for a given pit was smaller than the estimated minimum error (Fig. 1b).

\section{Conclusions}

In this work a research was carried out about the uncertainties on the corrosion pit depths at low magnifications and different tilting stage angles, by using SEM image stereo pairs from a Philips SEM 505 and the EZEImage stereo software. As a consequence of this study, the application on a corroded boiler tube piece of a power thermoelectric generator indicates that, although the uncertainty in the repeatability of the pit true shape may be large at some particular points, the calculations of their depths can be obtained with a smaller error. The obtained results in the case of pit depths in the $30-150 \mu \mathrm{m}$ range indicate that reliable values would be obtained by even working at a magnification as low as $100 \mathrm{X}$, although their errors remarkably diminish when magnifications of $200 \mathrm{X}$ and $400 \mathrm{X}$ and tilt angle $\Delta \phi= \pm 8^{\circ}$ are used.

The obtained results for orthopedic implant samples under two different surface treatments agree with the electrochemical results even though the conditions in both methods are very different, i.e, they do not generate pits with equal depth distributions. Nevertheless, more statistical work and more rigorous experimental conditions will be necessary for a better correlation among results for both superficial treatments. 


\section{Acknowledgements}

The authors acknowledge Mariela Theiller and Mario Sánchez of the Electron Microscopy

Laboratory of the CINDECA for technical support in the acquisition of SEM images. Also, they acknowledge the Consejo Nacional de Investigaciones Científicas y Técnicas (CONICET), Agencia Nacional de Promoción Científica y Tecnológica (ANCYPT) and Universidad Nacional de La Plata (UNLP) for financial support.

\section{References}

Aparicio, C., Gil, F. J., Fonseca, C., Barbosa, M., Planell, J.A. 2003. Corrosion behaviour of commercially pure titanium shot blasted with different materials and sizes of shot particles for dental implant applications. Biomaterials 24, 263-273.

API 570, 1998. Piping Inspection Code- Inspection, Repair, Alteration, and Rerating of InService Piping Systems, American Petroleum Institute Publication, second edition.

API 579, 2000. Fitness-for-Service, American Petroleum Institute Publication, first edition.

API 581, 2000.Risk Based Inspection, American Petroleum Institute Publication, Washington, DC, first edition.

Azar, V., Hashemi, B., Rezaee Yazdi, M. 2010. The effect of shot peening on fatigue and corrosion behavior of 316L stainless steel in Ringer's solution. Surf. Coat. Tech. 204, $3546-3551$.

Azevedo, C.R.F. and Marques, E.R. 2010. Three-dimensional analysis of fracture, corrosion and wear surfaces. Eng. Failure Anal. 17, 286-300. 
Bariani, P., De Chiffre, L., Hansen, H.N., Horsewell, A. 2005. Investigation on the traceability of three dimensional scanning electron microscope measurements based on the stereo-pair technique. Prec. Eng. 29, 219-228.

Barranco, V., Onofre, E., Escudero, M.L., García-Alonso, M.C. 2010. Characterization of roughness and pitting corrosion of surfaces modified by blasting and thermal oxidation. Surf. Coat. Tech. 204, 3783-3793.

Bonetto, R.D., Ladaga, J.L., Ponz, E. 2006. Measuring Surface Topography with Scanning Electron Microscopy. II. Analysis of Three Estimators of Surface Roughness in Second-dimension and Third-dimension. Microsc. Microanal. 12, (2), 178-186.

Chen, X., Ostadi, H., Jiang, K. 2010. Three-dimensional surface reconstruction of diatomaceous frustules. Anal. Biochem. 403, 63-66.

Choules, B., Metcalf, J. and Merk, J. 2009. Can a Critical Breakdown Potential be Established for Electrochemical Corrosion Testing of Medical Devices According to ASTM F2129?. Medical Device Materials V: Proceedings from the Materials \& Processes for Medical Devices Conference, Minneapolis, MN, USA, pp. 19-22.

Davies, P.A., Randle, V. 2001. Combined application of electron backscatter diffraction and stereo-photogrammetry in fractography studies. J. Microsc. 204, 29-38.

Drzazga, W., Paluszynski, J., Slowko, W. 2006. Three-dimensional characterization of microstructures in a SEM. Meas. Sci. Tech. 17, 28-31.

Fatikow, S., Dahmen, C., Wortmann, T., Tunnell, R. 2009. Vision Feedback for Automated Nanohandling. Proceedings of the 2009 IEEE International Conference on Information and Automation. Zhuhai/Macau, China, 806-811.

Heitz, E. 2006. Analytical Methods in Corrosion Science and Engineering, Edited by Philippe Marcus and Florian Mansfeld, USA, pp. 444. 
Hortolà, P. 2009. Using digital anaglyphy to improve the relief effect of SEM micrographs of bloodstains. Micron 40, 409-412.

Huang, Z., Dikin, D.A., Ding, W., Qiao, Y., Chen, X., Fridman, Y., Ruoff, R.S. 2004. Three-dimensional representation of curved nanowires. J. Microsc. 216, 206-214.

Ikeuchi, K., Horn, B.K.P. 1981. Numerical Shape from Shading and Occluding Boundaries. Artif. Intell. 17, 141-184.

Jahnisch, M. and Fatikow, S. 2007. 3-D vision feedback for nanohandling monitoring in a scanning electron microscope. International Journal of Optomechatronics, 1: 4-26.

Lane, G.S. 1969. The application of stereographic techniques to the scanning electron microscope. J. Phys. E: Sci. Instrum. 2, (7), 565-569.

Lane, G.S. 1972. Dimensional measurements. In Hearle, J.W.S., Sparrow, J.T. \& Cross, P.M. (Eds.). The Use of the Scanning Electron Microscope. Pergamon Press, New York, 219-238.

Malboubi, M., Ostadi, H., Wang, S., Gu, Y., Jiang, K. 2009. Effects of the Surface Morphology of Pipette Tip on Giga-seal Formation. Engineering Letters 17:4, (5pp).

Marinello, F., Bariani, P., Savio, E., Horsewell, A., De Chiffre, L. 2008. Critical factors in SEM 3D stereo microscopy. Meas. Sci. Technol. 19, 065705 (12pp).

Ostadi, H., Jiang, K., Prewett, P.D. 2009. Characterisation of FIB milling yield of metals by SEM stereo imaging technique. Microelec. Eng. 86, 1021-1024.

Ostadi, H., Jiang, K., Hukins, D.W.L. 2010. A comparison of surface roughness analysis methods applied to urinary catheters. Prec. Eng. 34, 798-801.

Paluszynski, J., Slówko, W. 2008. Measurements of the surface microroughness with the scanning electron microscope. J. Microsc. 233, 10-17. 
Pintus, R., Podda, S., Vanzi, M. 2005. Image alignment for 3D reconstruction in a SEM. Microelectronics Reliability 45, 1581-1584.

Pintus, R., Podda, S., Vanzi, M. 2008. An Automatic Alignment Procedure for a FourSource Photometric Stereo Technique Applied to Scanning Electron Microscopy. IEEE Trans. Instr. Meas. 57, (5), 989-996.

Pohler, O.E.M. 1986. Failures of Metallic Orthopedic Implants. In Failure Analysis and Prevention, Vol. 11, ASM Handbook, 9th ed., ASM International, USA, 670-694.

Ponz, E., Ladaga, J.L., Bonetto, R.D. 2006. Measuring Surface Topography with Scanning Electron Microscopy. I. EZEImage: A program to Obtain 3D Surface Data. Microsc. Microanal. 12, (2), 170-177.

Stampfl, J., Scherer, S., Gruber, M., Kolednik, O. 1996. Reconstruction of surface topographies by scanning electron microscopy for application in fracture research. Appl. Phys. A 63, 341-346.

Sun, C. 2002. Fast stereo matching using rectangular subregioning and 3D maximumsurface techniques. Inter. J. Comput. Vision 47, 99-117.

Tiansheng Wang, Jinku Yu, Bingfeng Dong. 2006. Surface nanocrystallization induced by shot peening and its effect on corrosion resistance of 1Cr18Ni9Ti stainless steel. Surface \& Coatings Technology 200, 4777-4781.

Vynnyk, T., Schultheis, T., Fahlbusch, T., Reithmeier, E. 2010. 3D-measurement with the stereo scanning electron microscope on sub-micrometer structures. J. Europ. Opt. Soc. Rapid Publications 5, 10038s-1-8.

Walker, C.G.H., El Gomati, M.M., Romanovsky, V. 2005. Reconstruction of images of surface height in scanning electron microscopy. Microscopy of Semiconducting materials: Proceedings of the 14th conference 107, 495-498. 
Wilde B.E. and Williams E. 1971. The use of current/voltage curves for the study of localized corrosion and passivity breakdown on stainless steel in chloride media. Electrochimica Acta 16, 1971-1985.

Wzorek, M., Czerwinski, A., Ratajczak, J., Dylewicz, R., Katcki, J. 2009. Selective etching of dislocations in GaN and quantitative SEM analysis with shape-reconstruction method. Micron 40, 37-40.

Wzorek, M., Czerwinski, A., Ratajczak, J., Lui, A., Iacob, E., Katcki, J. 2010. Depth measurements of etch-pits in GaN with shape reconstruction from SEM images. J. Microsc. 237, 242-245. 